\title{
Sacrificial Polymer Substrates in Photopolymerization-Based Micro 3D Printing for Fabrication and Release of Complex Micro Components
}

\author{
Vaut, Lukas; Zeng, Guanghong; Tosello, Guido; Boisen, Anja
}

Published in:

Advanced Materials Technology

Link to article, DOI:

10.1002/admt.201900378

Publication date:

2019

Document Version

Peer reviewed version

Link back to DTU Orbit

Citation (APA):

Vaut, L., Zeng, G., Tosello, G., \& Boisen, A. (2019). Sacrificial Polymer Substrates in PhotopolymerizationBased Micro 3D Printing for Fabrication and Release of Complex Micro Components. Advanced Materials Technology, 4(9), [1900378]. https://doi.org/10.1002/admt.201900378

\section{General rights}

Copyright and moral rights for the publications made accessible in the public portal are retained by the authors and/or other copyright owners and it is a condition of accessing publications that users recognise and abide by the legal requirements associated with these rights.

- Users may download and print one copy of any publication from the public portal for the purpose of private study or research.

- You may not further distribute the material or use it for any profit-making activity or commercial gain

- You may freely distribute the URL identifying the publication in the public portal 


\title{
Article type: Full Paper
}

\section{Sacrificial Polymer Substrates in Photopolymerization-based Micro 3D Printing for Fabrication and Release of Complex Micro Components}

\author{
Lukas Vaut*1, Guanghong Zeng, Guido Tosello and Anja Boisen*2
}

L.Vaut, Prof. A. Boisen

The Danish National Research Foundation and Villum Foundation's Center for Intelligent Drug Delivery and Sensing Using Microcontainers and Nanomechanics (IDUN), Department of Health Technology, Technical University of Denmark, Kgs. Lyngby, 2800, Denmark E-mail: ${ }^{1}$ lukv@dtu.dk, ${ }^{2}$ aboi@dtu.dk

Dr. G. Zeng, DFM A/S (Danish Fundamental Metrology), Hørsholm, 2970, Denmark

Prof. G. Tosello, Department of Mechanical Engineering, Technical University of Denmark, Kgs. Lyngby, 2800, Denmark

Keywords: micro additive manufacturing, sacrificial release layers, 3D microfabrication, micro stereolithography, micro medical device manufacturing

\section{Abstract Text}

$3 \mathrm{D}$ printing technology is widely employed in various scientific disciplines as well as

industrial applications such as hearing aid manufacturing. While technological advances and increasing resolution are making $3 \mathrm{D}$ printing accessible for microfabrication purposes, one question remains: how can small and delicate components like micro gears, lattices or micro medical devices be released from the build surface of the 3D printer without manual intervention? Herein, a method for 3D printing on top of water-soluble sacrificial substrates made from polyvinyl alcohol (PVA) is presented. Pre-fabricated sacrificial PVA substrates can be mounted onto a customized holder and serve as build surface during the $3 \mathrm{D}$ printing operation. The substrates do not only facilitate a mild release of 3D printed objects after dissolution of the sacrificial material, they also potentially allow for a convenient manipulation and further array-based processing of pre-determined patterns of printed structures subsequent to the $3 \mathrm{D}$ printing procedure. This, in turn, may enable a full integration into automated production lines. The fabrication of PVA substrates is thoroughly 
characterized and the 3D printing of various exemplary structures on sacrificial substrates is demonstrated. Finally, the release of 3D printed objects from PVA substrates is shown.

\section{Introduction}

3D printing has attracted interest since the release of the core inventions of stereolithography (SL) in 1986 and fused deposition modeling (FDM) in 1992, and continues to be a hot topic. ${ }^{[1,2]}$ The use of $3 \mathrm{D}$ printing spans a broad range of applications in different areas such as architecture, automotive industry and medicine. It is used to rapidly produce prototypes as well as functional end-products. Especially in the medical field, 3D printing holds great potential due to the possibility to fabricate customized components with high complexity.

Examples of already successful implementations of 3D printing in industrial fabrication of medical products comprise for example dental appliances and hearing aids. ${ }^{[3,4]}$ Medical and biomedical applications often require miniaturization of products. ${ }^{[5]}$ Due to advances in resolution and material availability, 3D printing has become a viable alternative to other microfabrication methods in many areas, including biomedical research. ${ }^{[6]}$ Research efforts in this area cover a broad variety of $3 \mathrm{D}$ printed prototypes, ranging from micro medical components such as bioresorbable vascular stents to microscale 3D scaffolds for tissue engineering, oral modified-release dosage forms as well as to propulsion-capable artificial microfish intended for toxin-neutralization applications. ${ }^{[7-10]}$

As pointed out by Quinlan et al., 3D printing, as of yet, has a low overall build rate when compared to other manufacturing processes, e.g. injection molding, and is therefore potentially less attractive for serial production. However, the start-up as well as maintenance costs related to conventional manufacturing processes like injection molding and machining can be very high, especially when the design complexity of the product increases. ${ }^{[11]}$ The low capital costs of 3D printing and its inherent flexibility thus makes it increasingly attractive for small to medium scale serial production in industry, for serial production of products that require mass customization (e.g. hearing aids), as well as for the rapid prototyping required in 
research and development. ${ }^{[12]}$ When compared to other lithography- or micromachining-based fabrication techniques, micro 3D printing is advantageous as the other techniques are limited with respect to three-dimensional complexity, ease of operation and production of assemblies with moving parts. ${ }^{[13]}$ One major drawback of 3D printing, however, is the low resolution compared to e.g. photolithography (minimum feature size of 2-3 $\mu \mathrm{m}$ ) and electron beam lithography (down to $5 \mathrm{~nm}$ ). ${ }^{[14]}$ It must be noted though, that $3 \mathrm{D}$ printing resolution is a subject of development and progress is shown on a frequent basis (e.g. custom built 3D printer by Gong et al. with a resolution of $7.6 \mu \mathrm{m}) .{ }^{[15]}$ Using common digital light processing (DLP)-based SL as well as conventional SL 3D printing, voxel sizes down to $30 \mu \mathrm{m}$ can easily be achieved.

Current micro 3D printing requires manual removal of the printed objects from the build surface by human intervention. This presents an obstacle towards automation and serial production as any component pattern enabling further computer numerical control (CNC) or other array-based processing is corrupted. Additionally, small prints are easily damaged during the manual print removal process. The release of single structures from a common substrate, such as a silicon wafer, by means of a sacrificial release layer, e.g. a water-soluble release layer, is a common procedure in micromachining and microfabrication. ${ }^{[16]}$ In FDM 3D printing, Polyvinyl alcohol (PVA) and high impact polystyrene (HIPS) are used as built-in sacrificial support structures.

Here, we use a rapidly exchangeable solid sacrificial material substrate as the build surface in vat photopolymerization-based $3 \mathrm{D}$ printing. The substrate enables further processing steps in an automated production line, and it allows for a mild release by dissolution of the sacrificial material. The working principle of the presented concept is that the solid sacrificial material substrate can be inserted into a vacuum actuated holder (Figure 1a). This assembly can be inserted into a desktop DLP-SL 3D printer, in which the PVA substrate serves as the build surface (Figure 1b). Later, the substrate can be utilized for easy manipulation of the 3D 
printed structures as well as for further processing steps. The PVA substrate can finally be dissolved in water to release the individual 3D printed structures. Advantages of PVA, in this case, include water solubility, chemical resistance against many solvents and biodegradability. ${ }^{[17]}$

\section{Results and Discussion}

\subsection{Compatibility Study using Raman Spectroscopy}

As a prerequisite for the presented concept to work, it is of importance to ensure that the contact between the substrate material and the liquid uncured 3D printing photopolymer does not alter the chemical status of the photopolymer and therefore not interfere with the photocuring reaction during the 3D printing procedure. Raman spectroscopy was performed and the spectra (molecular fingerprints) of untreated photopolymer as control and photopolymer after different durations of exposure to potential PVA contamination $(1 \mathrm{~h}, 3 \mathrm{~h}$, $1 \mathrm{~d}, 5 \mathrm{~d}$ ) compared (Figure 2). No changes could be observed and the PVA did not dissolve in the photopolymer or affect it otherwise. Consequently, we conclude that PVA does not alter the chemical status of the photopolymer, does not interfere with the $3 \mathrm{D}$ printing process and thus is a suitable substrate material. Supplementary experiments with further $3 \mathrm{D}$ printing photopolymers and PVAs support these findings and their results can be found in the supporting information (Figure S 1, Supporting Information).

\subsection{PVA Substrate Fabrication}

The substrates used in this work for vat photopolymerization $3 \mathrm{D}$ printing were fabricated by FDM 3D printing of a precursor substrate and subsequent compression molding (Figure 3a, b). This fabrication route has been chosen due to accessibility and increased flexibility with respect to the iterative nature of substrate fabrication optimization. Moreover, it demonstrates the successful fabrication at lab scale with most simple and affordable equipment. 
114 To demonstrate the possibility for cheap and scalable substrate production we also performed

115 laser cutting of a sheet of PVA (Figure 3c) as well as direct injection molding of PVA-

116 polymer substrates (Figure 3d).

\subsection{Geometrical Characterization of PVA Substrates}

118 For 3D printing, the fabricated PVA substrates must be produced with suitable surface

119 characteristics as well as uniform thickness. It is required that the substrates have sufficient

120 surface flatness to ensure good contact between the substrate and the polymerization interface

121 during the printing procedure, especially when the first layers of the objects are created. The peak-to-valley flatness parameter (FLTt; ISO 12781) was locally probed in areas of $1.27 \mathrm{x}$

$0.96 \mathrm{~mm}$ using optical profilometry with digital interferometry (DI) and confocal (CF)

124 observation conditions and analyzed after applying a robust gaussian filter (cut-off: $25 \mu \mathrm{m}$;

125 ISO 16610) to eliminate noise, outliers and short-wave details (Figure 4a). ${ }^{[18,19]}$ Different

126 substrates were analyzed: compression molded (CM), hand-roughened (CM-S) and injection

127 molded (IM) PVA substrates. A commercial anodized aluminum 3D printer build platform

128 (BP), plain aluminum substrates $(\mathrm{Al})$ and a silicon wafer $(\mathrm{Si})$ were included as reference

129 substrates. BP and Al substrates served to compare the fabricated PVA substrates to

130 frequently used 3D printing surfaces, Si exclusively served as a quality reference. The

131 analysis of the flatness measurements (Figure 5a) shows that, except for CM and Al samples,

132 which exhibit similar flatness (FLTt $\approx 2.4 \mu \mathrm{m}$; DI), samples have significantly different FLTt

133 values with large effect sizes (Table S 1, Supporting Information). During the compression

134 molding, the polymer surface adapts the negative of the molds' surface texture. Thus, CM

135 and Al samples have similar flatness as CM substrates were molded with the use of flat

136 aluminum sheets. While BP has the lowest flatness (FLTt $\approx 12.11$ (DI) and $15.55 \mu \mathrm{m}(\mathrm{CF})$ ),

137 Si has the highest flatness with an FLTt value $(0.18 \mu \mathrm{m}$; DI $)$ up to two orders of magnitude

138 lower than the ones of the other samples. In comparison with CM samples (FLTt $\approx 3.37 \mu$ m;

$139 \mathrm{CF}), \mathrm{CM}-\mathrm{S}$ samples show a reduced flatness (FLTt $\approx 5.46 \mu \mathrm{m} ; \mathrm{CF})$, which can be explained 
140 by the hand-roughening treatment as sanding marks can be observed (Figure 4a). IM samples

141 also show lower flatness $(5.78 \mu \mathrm{m}$; DI) when compared to CM. In the case of IM samples, the

142 surface texture is determined by the manufacturing of the molding tool.

143 The roughness of a surface can affect adhesion and has been found to be associated with the

144 bond strength of adhesives. In this regard, Shahid et al. found that the average roughness ( $\mathrm{Ra}$ )

145 is linearly correlated with average cleavage strength of steel/adhesive/steel cleavage

146 specimens, probably due to an increase in effective surface area and through the formation of

147 "mini scarf joints on adherend surfaces at micro level". ${ }^{[20]}$ Likewise, the successful use of

148 surface roughening treatments for increased adhesive bonding of titanium to polymer

149 composites has been thoroughly discussed. ${ }^{[21]}$ During the $3 \mathrm{D}$ printing procedure, it is

150 fundamental that the first layer of cured photopolymer adheres well to the build surface since

151 the 3D printed objects are subject to tensile stress due to continuous movement of the Z-axis

152 and subsequent separation from the polymerization interface. The local surface roughness,

153 more specifically the arithmetical mean height (Sa), was determined using digital

154 interferometry-based optical profilometry (Figure 4b). ${ }^{[22]}$ The evaluation of conducted Sa

155 measurements (Figure 5b) show significant differences with large effect sizes between the

156 different samples, except for CM-S $(\mathrm{Sa} \approx 573 \mathrm{~nm})$ and IM samples $(\mathrm{Sa} \approx 623 \mathrm{~nm})$ (Table S 2,

157 Supporting Information). Si has the lowest roughness $(\mathrm{Sa} \approx 2 \mathrm{~nm})$, which matches the

158 specifications of the manufacturer, while BP appears to have the roughest surface ( $\mathrm{Sa} \approx 1.79$

$159 \mu \mathrm{m})$. When compared to $\mathrm{BP}, \mathrm{CM}$ has a significantly lower roughness $(\mathrm{Sa} \approx 134 \mathrm{~nm})$. The

160 hand-roughening treatment is seen to greatly increase the roughness of CM-S substrates when

161 compared with CM substrates, which can also be seen in the example of the very complex

162 surface in Figure 4b. Even though CM-S and IM have similar Sa values, the surfaces exhibit

163 very different surface morphologies. The Sa value does not give any indications of the surface

164 morphology and therefore we calculated the developed interfacial area ratio (Sdr), which is a

165 measure for surface complexity and also a better indication of adhesive properties (Figure 
$1665 \mathrm{c}) .{ }^{[23]}$ The analysis of Sdr values shows significant differences between all samples (Table S

167 3, Supporting Information). The results mainly follow the trend that could be observed in Sa 168 measurements, with better differentiated values for CM-S and IM. The Sdr-value for CM-S

169 was two orders of magnitude higher than for CM.

170 Thickness measurements of different PVA substrates were conducted (Figure 5d), and the

171 measurements on deviation from target thickness show that values obtained for the

172 compression molded PVA substrates $(\mathrm{CM})$ lie in a range of $\approx 26 \mu \mathrm{m}$. In the case of hand-

173 roughened compression molded samples (CM-S) and injection molded samples (IM), the

174 measurements lie in a range of $\approx 43 \mu \mathrm{m}$ and $\approx 23 \mu \mathrm{m}$, respectively. To ensure a successful

175 printing without the need for recurring calibrations, the thickness deviation of the substrates

176 should be smaller than the layer thickness of the individually exposed layers during the 3D

177 printing procedure. As the layer height of the $3 \mathrm{D}$ printer in this case was $25 \mu \mathrm{m}$, a thickness

178 deviation above $25 \mu \mathrm{m}$ could call for recurring homing calibrations. The lack of precision in

179 thickness repeatability for CM substrates can partially be explained by the deviation in

180 material dispensing during FDM 3D printing of the precursor substrate. Here, an observed

181 weight deviation with a range of $8.66 \mathrm{mg}(\mathrm{N}=10)$ can translate into a $16-17 \mu \mathrm{m}$ thickness

182 deviation when taking the final substrate dimensions into account and assuming a PVA

183 density of 1.19-1.31 $\mathrm{g} \mathrm{cm}^{-3} \cdot{ }^{[24]}$ Furthermore, the manual handling during the molding

184 procedure leaves room for error. It is to be expected that the thickness deviation is higher for

185 CM-S substrates than for CM substrates, since it is likely that the hand-roughening treatment

186 unevenly affected the final thickness of the substrates. We note that the deviation for CM

187 substrates is not much higher than for injection molded substrates. A further optimization of

188 the CM fabrication processes can lead to a much higher precision in thickness repeatability,

189 allowing for users without access to injection molding to fabricate their own high-quality

190 substrates. 
191 Since standard deviations were smaller than $\pm 25 \mu \mathrm{m}$ in all cases, the study was continued

192 based on the same CM fabrication process and without recurring homing calibrations.

\subsection{D Printing on PVA Substrates}

194 Using a commercial DLP-SL 3D printer and a custom vacuum-actuated holder (see Figure 1a),

195 we were able to 3D print various exemplary structures on CM PVA substrates (Figure 6). The

196 workflow allowed us to 3D print arrays of defined geometrical objects on top of PVA

197 substrates and to remove the entire substrate from the holder after the finished 3D printing 198 operation. 3D printed example structures include those, e.g. helical micro-gear and micro-

199 truss lattice, which are nearly impossible to fabricate by other conventional manufacturing 200 techniques, such as injection molding or micromachining.

201 Employing a specifically for this purpose designed and 3D printed test object (Figure 6h) and 202 a texture analyzer, we determined the detachment force to study the relationship between surface characteristics of the build surface and bond strength of the 3D print. In this regard,

204 we interpret the detachment force to be proportional to the bond strength as a higher

205 detachment force is caused by a higher bond strength. The footprint of the test object matches 206 the dimensions of areas probed for the flatness characterization. Arrays of the test object were 207 3D printed on BP, Al, CM and CM-S substrates (Figure 7a, b, c) and using a customized texture analyzer setup (Figure 7d), detachment force as well as work of adhesion (area under curve of detachment graph) were determined (Figure 7e). The evaluation of the detachment

210 force (Figure 7f) shows statistically significant differences with large effect sizes between all

211 samples (Table S 4, Supporting Information). Hand-roughening of PVA substrates

212 significantly affected the bond strength between the test objects and CM-S substrates, thus

213 revealing a much higher detachment force when compared to CM substrates. Despite having a

214 rougher surface, $\mathrm{Al}$ and $\mathrm{BP}$ have lower detachment forces while $\mathrm{Al}$ has the lowest. An

215 explanation for this might be the influence of other adhesion promoting factors, which could

216 lead to the occurrence of increased polymer-polymer (cured photopolymer-PVA) interactions. 
217 An example for those could be electrostatic adhesion between the PVA substrates and the 3D

218 printed objects according to the electrostatic theory of adhesion, hence leading to a higher

219 bond strength. ${ }^{[25]}$ This possible explanation is consistent with the observation that the

220 detached 3D printed objects kept sticking to other objects, e.g. the custom microgripper after

221 they have been detached from the PVA substrates. The evaluation of the work of adhesion

222 (Figure $7 \mathrm{~g}$ ) follows a similar trend, except for the fact that no significant difference between

$223 \mathrm{BP}$ and $\mathrm{Al}$ can be found (Table S 5, Supporting Information).

224 The results indicate that the surface of the proposed PVA substrates may be tailored for

225 optimized performance, e.g. through a surface roughening treatment for increased adhesion of

226 the 3D printed structures to the substrate. However, as already noted in the case of potential

227 influence of electrostatic adhesion, there are most probably further parameters, which can

228 positively or negatively influence the adhesive bond (e.g. surface chemistry and interfacial

229 failure). Moreover, the formation of the adhesive bond itself does not represent the only

230 potential influence that is relevant for a successful 3D printing outcome. As mentioned earlier,

231 the adhesion of 3D printed objects to the substrate was considered to be crucial as the freshly

232 printed layers are subject to tensile stresses caused by the vertical motion of the build platform

233 and the repetitive contact to and separation from the polymerization interface. It is to be

234 expected that the same tensile stresses act on the PVA substrate as well and they could

235 potentially lead to deformation or detachment of the entire PVA substrate. Although these

236 issues could not be detected during the experiments, the different forms of mechanical stress

237 and the consequences they might have should be considered with regards to the potential

238 setting and application of the proposed method.

2.5. Release of 3D Printed Objects from PVA Substrates

240 An array of helical micro-gears (Figure 6e) 3D printed on CM PVA substrates was released

241 from the substrate within 150 min. (Figure 8a; Movie S 1, Supporting Information). Scanning

242 electron microscopy of the harvested individual micro-gears shows that the gears are intact 
and free of substrate material (Figure $8 \mathrm{~b}, \mathrm{c}$ ). The dissolution rate of PVA is highly dependent

244 on the type of PVA (degree of polymerization, degree of hydrolysis) and also on the

245 temperature. ${ }^{[16]}$ Furthermore, the time needed for the dissolution depends on the amount of

246 material to be dissolved. In Figure 8a it is visible that the PVA substrate was inserted into the

247 dissolution medium in a 90-degree orientation. Since, upon contact with water, the PVA

248 started to dissolve and became rather jelly-like, the substrate lost its mechanical rigidity and

249 deformed. The latter can be observed as a progressing trend within the first 90 minutes. At $\mathrm{t}=$

25090 min some individual released micro-gears were visible, whereas the remnants of the

251 substrate transformed into a clot and engulfed all other micro-gears that were still in contact

252 with the PVA. As a consequence, the PVA needed to be fully dissolved in order to release all

253 3D printed micro-gears, which accounts for the 150 min release time.

254 To illustrate that the release time can be reduced, composite CM PVA substrates with a non-

255 dissolving polylactic acid (PLA) core were fabricated. The PLA core was fully encapsulated

256 by the surrounding PVA and reduced the total amount of PVA by $50 \%$. Using this substrate,

257 the same array of micro-gears could be released within 90 min. (Figure S 2, Movie S 2,

258 Supporting Information). In this case, the non-dissolving PLA core helped to largely maintain

259 the mechanical rigidity of the substrate, so that a clot formation was impossible and only the

260 PVA interfacing the 3D printed micro-gears and the PLA core had to dissolve in order to

261 release all micro-gears. It has to be emphasized that the two experiments are only examples of

262 how the release process could look like, as many influential parameters, such as type and

263 properties of the utilized PVA, have not been investigated. Beyond the dissolution properties

264 of the PVA, factors like excitation type (ultrasound, stirring, flow etc.) and substrate porosity

265 might have a positive influence on diffusion and convection of the dissolving PVA and

266 therefore on the dissolution time. A further optimization of the release procedure can be

267 expected to drastically reduce the required dissolution time. 


\section{Conclusion}

270 In summary, we have demonstrated the use of water-soluble PVA sacrificial substrates in vat 271 photopolymerization-based 3D printing. The fabrication of substrates with suitable flatness, 272 roughness and thickness characteristics was accomplished at lab scale, and their specifications 273 are compatible with industrial fabrication. The substrates were chemically compatible with 274 different 3D printing photopolymers and exhibited good bond strengths to the 3D printed 275 objects. Using a custom-made vacuum-actuated holder, PVA substrates could be rapidly exchanged and taken from the 3D printer, thereby enabling further array-based processing and potential integration into automated production lines. We showed that advanced 3D printed objects can be released through dissolution of the substrate, thereby eliminating the need for manual intervention. Consequently, the proposed method might be potent of promoting the

280 application of 3D printing for the serial production of complex micro components.

\section{Experimental Section}

282 Materials: All chemicals and reagents were used as received. For the fabrication of PVA

283 substrates different kinds of PVA material were used: RS Pro PVA 3D printing filament (RS

284 Components A/S, Denmark), MOWIFLEX ${ }^{\mathrm{TM}} \mathrm{C} 17$ and MOWIFLEX ${ }^{\mathrm{TM}} \mathrm{C} 600$ (Kuraray Nordic 285 Ab Oy, Finland). HTM 140M V2 3D printing photopolymer (EnvisionTEC GmbH, Germany) 286 was used to 3D print onto PVA substrates. Further photopolymers were used for a compatibility study: PIC100 (EnvisionTEC GmbH, Germany) and Form Clear resin

288 (Formlabs GmbH, Germany). 2-propanol (Sigma-Aldrich Denmark A/S, Denmark) was used 289 for the post-treatment of 3D printed structures.

290 Compatibility study using Raman spectroscopy: A compatibility assay was performed by 291 incubating $200 \mathrm{mg}$ of solid polyvinyl alcohol (PVA) material in $1 \mathrm{ml}$ of liquid 3D printing 292 photopolymer and analyzing a sample of the liquid after successive timepoints $(1 \mathrm{~h}, 3 \mathrm{~h}, 1 \mathrm{~d}, 5$ 293 d) by Raman spectroscopy. When considering the use of one PVA substrate with a weight of $294805 \mathrm{mg}$ in the supplied vat of the 3D printer, which is filled with $150 \mathrm{ml}$ of 3D printing 
polymer, the concentration amounts to $5.37 \mathrm{mg} \mathrm{ml}^{-1}$. The ratio of PVA to 3D printing polymer 296 in the compatibility study was chosen to be multiple times higher. 3D printing polymer which 297 was not in contact with PVA served as control. Raman spectroscopy was employed to 298 determine molecular fingerprints of the samples.

299 Raman spectra were acquired with an in-house-built Raman spectroscopy system with 300 improved sensitivity for Raman scattering registration in case of liquid samples. The system is 301 based on a high power $(500 \mathrm{~mW})$ multimode laser with a wavelength of $785 \mathrm{~nm}$. The laser 302 had an intensity of $20 \mathrm{~mW} \mu \mathrm{m}^{-2}$ and was focused on the sample through a liquid container 303 with a $\mathrm{CaF}_{2}$ bottom plate. Measurements were carried out with a spectral resolution of $1.8 \mathrm{~cm}^{-}$ $304{ }^{1}$ in the range from 350 to $2100 \mathrm{~cm}^{-1}$ and collected using a CCD sensor. Wavelength and 305 spectral sensitivity calibration of the instrument was performed according to ASTM 1840 and 306 ASTM E2911 international guidelines.

307 Fabrication of PVA substrates: Whereas the FDM-3D printing step did not serve to produce 308 the final substrate, but rather as a material dispensing step to fabricate a precursor substrate of 309 a certain size, the compression molding process acted to transform the precursor into a flat 310 substrate of desired shape by using a mold assembly. For the fabrication of the substrate 311 precursor, a commercially available Original Prusa i3 MK2S desktop 3D printer was used 312 (Prusa Research, Czech Republic) to print with likewise commercially acquired RS Pro PVA 313 filament with a $100 \%$ infill, a hotend temperature of $210{ }^{\circ} \mathrm{C}$ and a print bed temperature of $31485{ }^{\circ} \mathrm{C}$ (first layer) and $60^{\circ} \mathrm{C}$ (following layers). The volume of the substrate precursor was 315 calculated to equal the volume of the mold cavity which is used in the compression molding 316 step. While the FDM 3D printing method can be quite accurate, it is - due to the nature of this 317 technology - not precise enough to exactly dispense the correct volume of material as the 318 layer-by-layer and line-by-line fabrication leads to the creation of small gaps within the print 319 even though the infill ratio is set to $100 \%$. In order to compensate for this phenomenon, the 320 volume of the substrate precursor was increased by $3 \%$, which was found to be an acceptable 
value to obtain a good substrate after compression molding. After FDM 3D printing of the precursor substrates, the substrates had an average weight of $804.96 \mathrm{mg}(\mathrm{N}=10)$, ranging from 800.09 to $808.75 \mathrm{mg}$ with a standard deviation of $2.93 \mathrm{mg}$.

The mold assembly for the compression molding consisted of a $1 \mathrm{~mm}$ thick aluminum mold, $90 \mu \mathrm{m}$ aluminum foil and $1 \mathrm{~mm}$ stainless steel sheets. The compression molding procedure was carried out with a pressure of $55 \mathrm{kN}$ and a temperature of $160^{\circ} \mathrm{C}$ using a PW-H HKP300 laboratory press (Paul-Otto Weber GmbH, Germany).

For some of the resulting substrates, the surface was modified by sanding one side with 600

329 grit sanding paper.

330 Composite compression molded substrates consisting of PVA and polylactic acid (PLA) were 331 as well fabricated using an FDM 3D printing and a compression molding step. PLA inserts 332 were $3 \mathrm{D}$ printed with smaller dimensions, constituting $50 \%$ of the final substrate. PVA 333 substrates were designed to have a cavity and the 3D printing procedure was paused as soon 334 as the cavity was completed. Then the PLA insert was inserted into the cavity and the 3D 335 printing procedure was continued. The cavity was closed with the remaining layers of PVA, 336 thereby fully engulfing the PLA in its' core. The compression molding step transformed the 337 precursor composite substrates into smooth PLA-PVA core-shell substrates of $1 \mathrm{~mm}$ thickness 338 using the same conditions as with plain PVA substrates.

339 In a different approach, a Press 300 SV laboratory platen press (Dr. Collin GmbH, Germany) 340 served to transform $15 \mathrm{~g}$ of MOWIFLEX ${ }^{\mathrm{TM}}$ C17 PVA polymer pellets into a compressed 341 sheet using a pressure of $50 \mathrm{bar}$ and a temperature of $150{ }^{\circ} \mathrm{C}$ for a duration of $1000 \mathrm{~s}$ and 342 subsequently cooling it down to $30{ }^{\circ} \mathrm{C}$ within 500 s. Substrates of desired shape were cut from 343 the sheet with an Epilog Mini 18 laser cutter (Epilog Laser BV, The Netherlands) which was 344 equipped with a $30 \mathrm{~W} \mathrm{CO}_{2}$ laser. This procedure needed to be performed with the necessary 345 safety precautions as toxic fumes can be release during the procedure. ${ }^{[24]}$ 
Injection molding of PVA substrates was performed using an Arburg Allrounder 370A injection molding machine (Arburg GmbH \& Co KG, Germany) equipped with an $18 \mathrm{~mm}$ screw and MOWIFLEX ${ }^{\mathrm{TM}}$ C600 PVA polymer. Injection molding parameters were adjusted to 70 bar back pressure, $180{ }^{\circ} \mathrm{C}$ melt temperature, $40{ }^{\circ} \mathrm{C}$ mold temperature, $50 \mathrm{~mm} \mathrm{~s}^{-1}$ injection velocity, 500 bar packing pressure, $10 \mathrm{~s}$ packing time and $40 \mathrm{~s}$ cooling time. Characterization of PVA and reference substrates: The thickness of the fabricated substrates was measured in the center and in the four corners of each substrate using an RS Pro micrometer screw with an error of $0.001 \mathrm{~mm}$ (RS Components, Denmark). A PLu neox optical 3D profiler (Sensofar Metrology, Spain) served to conduct surface topology measurements, using confocal and interferometric microscopy. To analyze the flatness property of the various specimen, 10X interferometry and 20X confocal lenses were used for data acquisition. To compensate the loss in field of view when using the $20 \mathrm{X}$ confocal lens, stitching was used to combine four images to one bigger area image. A $50 \mathrm{X}$ interferometry lens was used to acquire data for the analysis of the surface roughness. In case of all specimens, a sampling procedure based on a $20 \times 20 \mathrm{~mm}$ grid was performed to obtain surface 361 measurements in a total of 25 spots in always the same relative positions. A 3" silicon wafer 362 (No. 16013, Ted Pella inc., USA) with a specified roughness and total thickness variation of 2 $\mathrm{nm}$ and $<20 \mu \mathrm{m}$, respectively, as well as the supplied build platform of an EnvisionTec Micro

364 Plus High-Res DLP 3D printer were used as reference surfaces. Treatment and analysis of surface metrology data was done in SPIP 6.7.4 (Image Metrology A/S, Denmark) analytical software.

367 Computer aided design (CAD): All design tasks were carried out using SolidWorks 2015 368 (Dassault Systèmes SolidWorks Corporation, USA) and OpenSCAD open source software. Machining of customized 3D printer build platform: A customized 3D printing build platform

370 featuring a four-point spring leveling mechanism and a vacuum-actuated holding cavity for a 371 print substrate was made to retrofit a Micro Plus High-Res digital light processing (DLP) 3D 
printer (EnvisionTec GmbH, Germany). The platform was machined by an external machining shop using a combination of CNC milling and electrical discharge machining. 3D printing on PVA substrates: The 3D printing on PVA substrates was conducted with an EnvisionTec Micro Plus High-Res DLP 3D printer with a XY resolution of $30 \mu \mathrm{m}$ pixel size and a $\mathrm{Z}$ resolution of $25 \mu \mathrm{m}$. The $3 \mathrm{D}$ printer was retrofitted with a customized build platform to enable a flush leveling of the platform to the polymerization interface of the printer.

378 Perfactory RP software (EnvisionTEC GmbH, Germany) served to create print files from the prepared CAD models. After the printing procedure, the PVA substrate with printed structures on top was first cleaned from excess printing material in a beaker with 2-propanol placed in an ultrasound bath for 5 min and subsequently post-cured in an UV oven for $10 \mathrm{~min}$

382 (EnvisionTEC GmbH, Germany).

383 Scanning electron microscopy: All scanning electron microscopy was performed using a TM3030Plus tabletop scanning electron microscope (Hitachi High Technologies Europe GmbH, Germany). A 208HR high resolution sputter coater (Cressington Scientific Instruments, UK) equipped with a gold target was used to coat the specimens with a thin layer

387 of gold $(\approx 20 \mathrm{~nm})$ prior to observation.

388 Determination of detachment force: A TA.XT plus Texture Analyzer (Stable Micro Systems, 389 UK) equipped with a $10 \mathrm{~kg}$ load cell and a customized probe was used to measure detachment

390 forces needed to separate a printed sample from different 3D printing substrates. Detachment

391 forces and work of adhesion were computed with a customized python program.

392 Release of micro 3D prints from PVA substrates: 3D printed structures were released from the 393 PVA substrate by retaining the substrate in a small box with a bottom of fine stainless-steel mesh and placing it in a de-ionized water-filled beaker, which in turn was placed into an

395 ultrasound bath at a temperature of $55^{\circ} \mathrm{C}$. The samples were kept in the ultrasound bath until 396 all PVA was dissolved. A waterproof USB endoscopic camera and Video Velocity Free 397 software (Candy Labs Media, Canada) were used to record time-lapse photos during the 
398 release procedure. The samples were ultimately taken out of the water and left to dry in an 399 oven at $37^{\circ} \mathrm{C}$.

400 Statistics: All presented statistics were computed using R programming language and RStudio 401 software (RStudio Inc., USA) as well as Microsoft Excel (Microsoft Corporation, USA). As 402 in case of reference samples Si and BP only one specimen was available each, t-test results 403 comparing those with Alu, CM, CM-S and IM samples are based on the assumption that the 404 measured reference samples constitute ideal and representative samples of their kind. The 405 results obtained in these cases can serve as an indication only, because resulting p-values 406 might be distorted. Consequently, the reported effect sizes (Hedges' g) are more reliable.

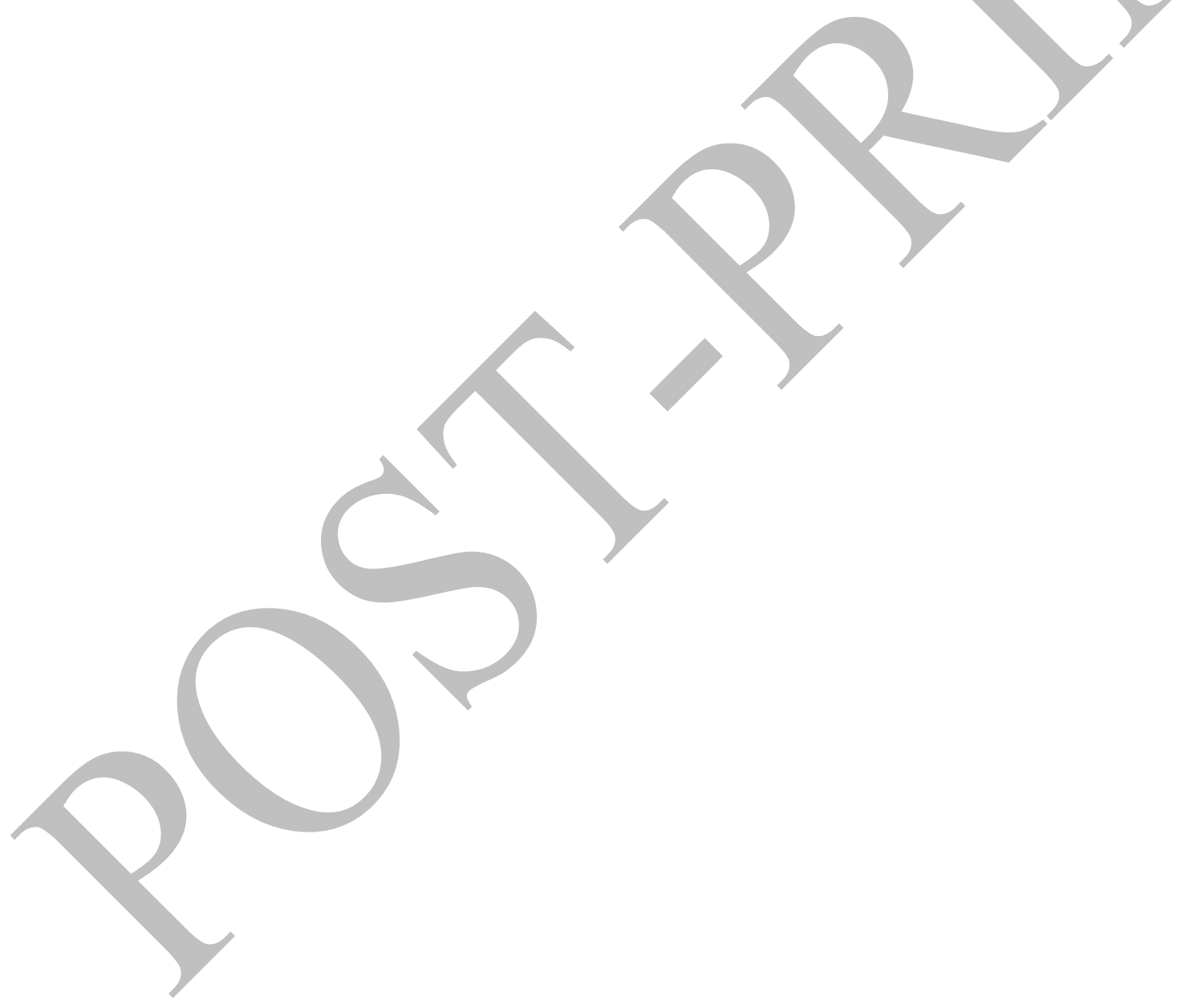


Supporting Information is available from the Wiley Online Library or from the author.

\section{Acknowledgements}

411 The authors would like to acknowledge the Center for Intelligent Drug Delivery and Sensing 412 Using Microcontainers and Nanomechanics (IDUN) whose research is funded by the Danish National Research Foundation (DNRF122) and Villum Fonden (Grant No. 9301).

Dr. Guanghong Zeng acknowledges funds from the Danish Agency for Institutions and Educational Grants, metrology for additively manufactured medical implants (short name: MetAMMI, project number: 15HLT09) from the EMPIR program. The EMPIR program is co-financed by the participating states and the European Union's Horizon 2020 research and innovation program.

The authors would also like to thank Dr. Kristian E. Jensen and Dr. Tommy Sonne Alstrøm for help with the manuscript preparation, Francesco Regi for performing injection molding and Jesper Scheel for photography. Furthermore, the authors would like to thank Ann-Britt Aspholm-van der Brugge and Kuraray Nordic Ab Oy for straightforward communication, great service and provision of free polymer samples.

\section{Conflict of Interest}

The authors declare no conflict of interest.

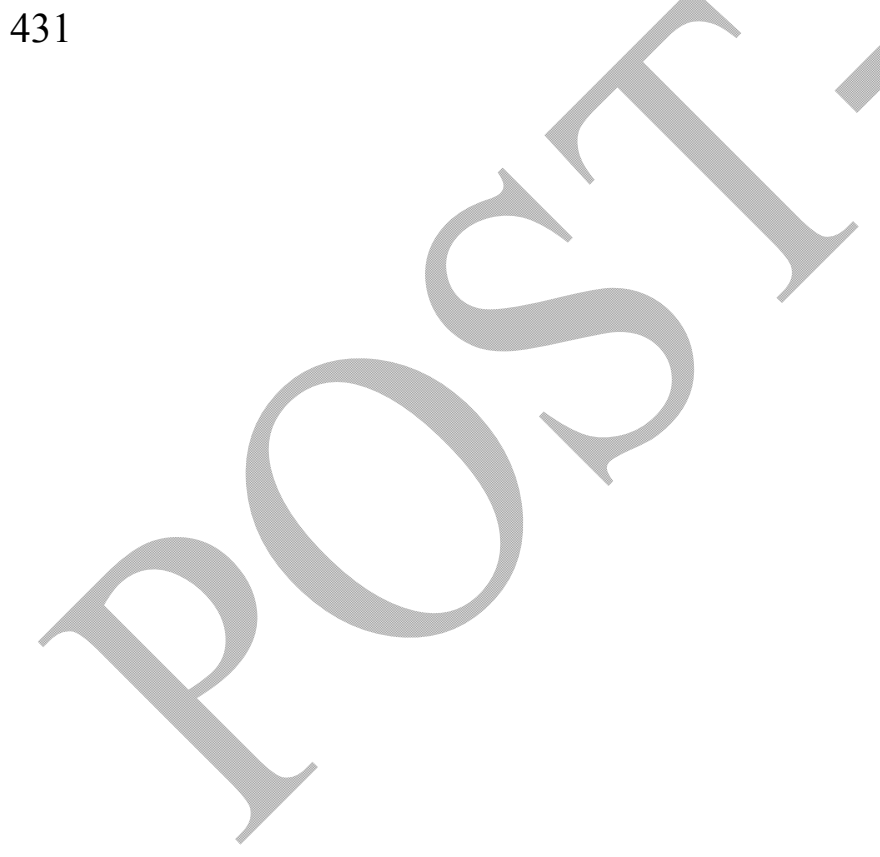


433 [1] C. W. Hull (UVP Inc.), US 45753301986.

$434 \quad$ [2] S. S. Crump (Stratasys Inc.), US 51213291992.

435 [3] N. Hopkinson, R. J. M. Hague, P. M. Dickens, An Industrial Revolution for the Digital Age, Wiley, Hoboken, NJ, USA, 2006.

437 [4] C. G. Sandström, Technol. Forecast. Soc. Change 2016, 102, 160.

438 [5] L. Alting, F. Kimura, H. N. Hansen, G. Bissacco, CIRP Ann. 2003, 52, 635.

439 [6] B. Gross, S. Y. Lockwood, D. M. Spence, Anal. Chem. 2017, 89, 57.

440 [7] R. van Lith, E. Baker, H. Ware, J. Yang, A. C. Farsheed, C. Sun, G. Ameer, Adv. Mater. Technol. 2016, 1,1 .

442 [8] N. A. Chartrain, C. B. Williams, A. R. Whittington, Acta Biomater. 2018, 74, 90.

443 [9] J. Wang, A. Goyanes, S. Gaisford, A. W. Basit, Int. J. Pharm. 2016, 503, 207.

444 [10] W. Zhu, J. Li, Y. J. Leong, I. Rozen, X. Qu, R. Dong, Z. Wu, W. Gao, P. H. Chung, J. Wang, S. Chen, Adv. Mater. 2015, 27, 4411.

446 [11] H. E. Quinlan, T. Hasan, J. Jaddou, A. J. Hart, J. Ind. Ecol. 2017, 21, S15.

447 [12] C. Kapetaniou, A. Rieple, A. Pilkington, T. Frandsen, P. Pisano, Technol. Forecast. Soc. Change 2018, 128, 22.

449

[13] M. Vaezi, H. Seitz, S. Yang, Int. J. Adv. Manuf. Technol. 2013, 67, 1721.

450 [14] A. Pimpin, W. Srituravanich, Eng. J. 2012, 16, 37.

451 [15] H. Gong, B. P. Bickham, A. T. Woolley, G. P. Nordin, Lab Chip 2017, 17, 2899.

452 [16] V. Linder, B. D. Gates, D. Ryan, B. A. Parviz, G. M. Whitesides, Small 2005, 1, 730.

453 [17] C. A. Finch, Polyvinyl Alcohol, Properties and Uses, Wiley, Hoboken, NJ, USA, 1973.

454 [18] ISO 12781-1:2011 - Geometrical Product Specifications (GPS) -- Flatness -- Part 1:

455 Vocabulary and Parameters of Flatness, International Organization For

456 Standardization, Vernier, Geneva, Switzerland, 2011.

457 [19] ISO 16610-71:2014 - Geometrical Product Specifications (GPS) -- Filtration -- Part 
71: Robust Areal Filters: Gaussian Regression Filters, International Organization For Standardization, Vernier, Geneva, Switzerland, 2014.

460

[20] M. Shahid, S. A. Hashim, Int. J. Adhes. Adhes. 2002, 22, 235.

461 [21] P. Molitor, V. Barron, T. Young, Int. J. Adhes. Adhes. 2001, 21, 129.

[22] ISO 25178-2:2012 - Geometrical Product Specifications (GPS) -- Surface Texture: Areal -- Part 2: Terms, Definitions and Surface Texture Parameters, International Organization For Standardization, Vernier, Geneva, Switzerland, 2012.

[23] R. Leach, Characterisation of Areal Surface Texture, Springer Berlin Heidelberg, Berlin, Heidelberg, 2013.

467 [24] ILO, WHO, "ICSC 1489 Polyvinyl alcohol," https://www.ilo.org/dyn/icsc/showcard.display?p_lang=en\&p_card_id=1489\&p_versio

[25] W. C. Wake, Polymer (Guildf). 1978, 19, 291.

[26] L. Rose, "Internal Structures for SLA, DLP, or SLS style printing," https://www.thingiverse.com/thing:1394337/files, accessed: 03, 2018. $\mathrm{n}=2$, accessed: 09, 2018 . 
a

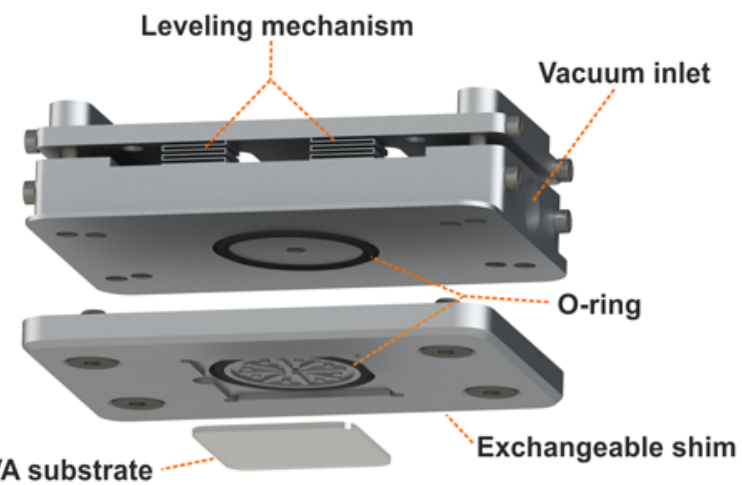

b

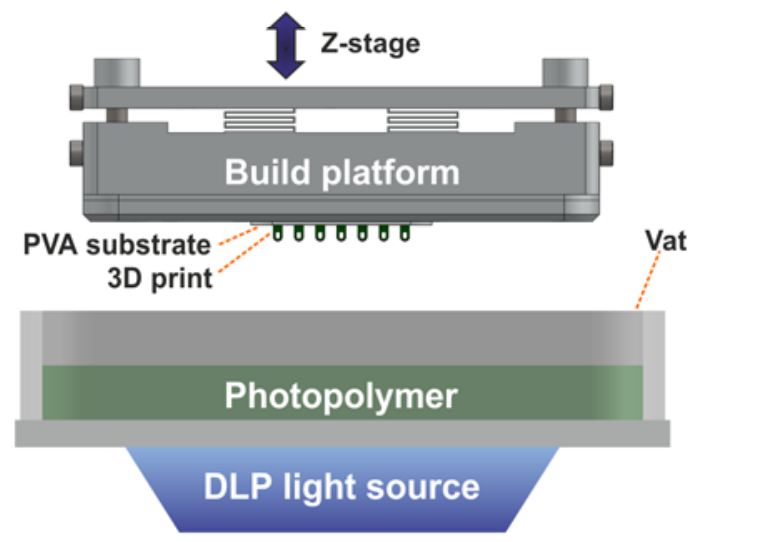

Figure 1. a) Design of customized vacuum-actuated substrate holder for the use in a desktop DLP-SL 3D printer. b) Schematic illustration of working principle of using pre-fabricated PVA substrates in a DLP-SL 3D printer. The PVA substrate is used as the build surface and held in place by the vacuum-actuated holder (build platform) which moves in $\mathrm{Z}$ direction. In an industrial production line setting, the holder could be operated by a robotic arm which also carries out further processing steps. 


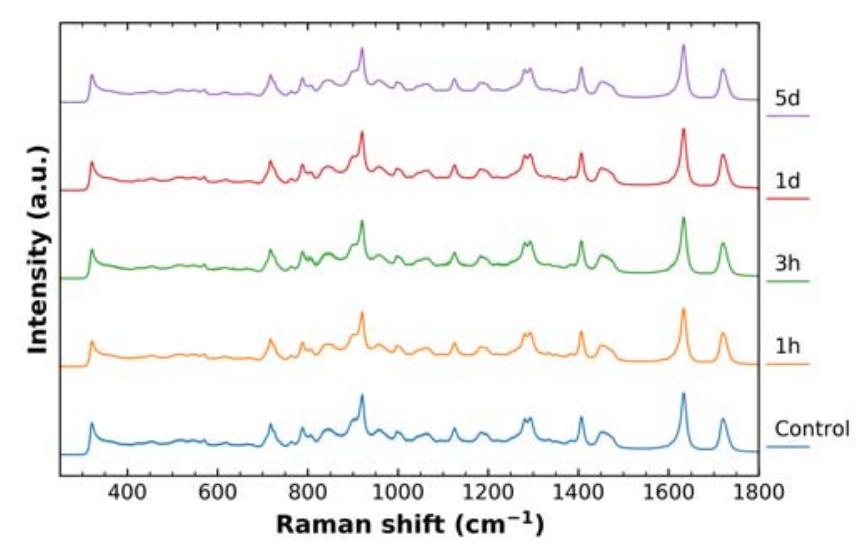

Figure 2. Molecular fingerprint of HTM 140 M 3D printing photopolymer after contact with 486 RS Pro PVA filament for different time durations ( $1 \mathrm{~h}, 3 \mathrm{~h}, 1 \mathrm{~d}$ and $5 \mathrm{~d})$ and untreated 
a
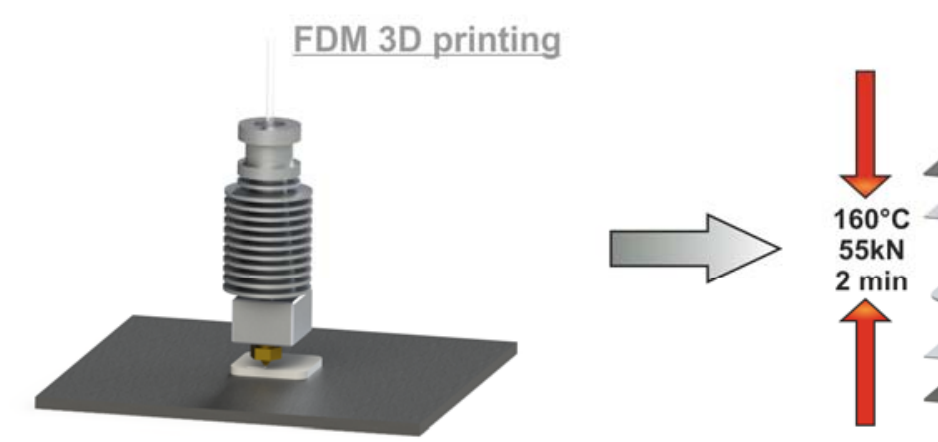

Compression
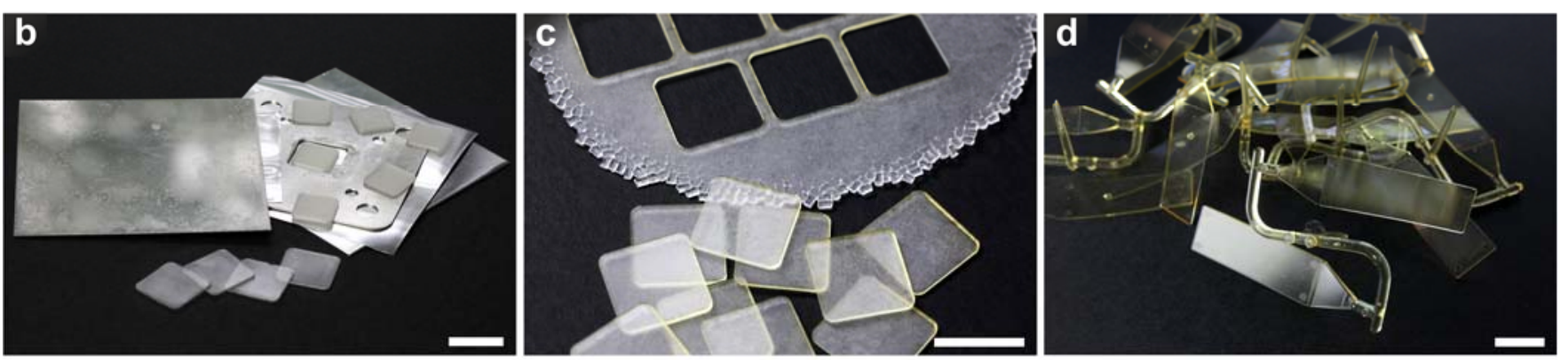

489

490

Figure 3. PVA substrate fabrication. a) Schematic illustration of two-step PVA substrate

491

492

493

fabrication sequence using fused deposition modelling (FDM) 3D printing and subsequent compression molding. b-d) Photographs of differently fabricated PVA substrates. b) FDM 3D printed precursor substrates (substrates placed on mold assembly) and compression molded

494 substrates (front). c) Laser-cut substrates from compressed PVA sheet. d) Injection molded PVA substrates in standard object slide format. Scale bars are equal to $25 \mathrm{~mm}$.
3D printed

Aluminum mold

Aluminum foil

Steel plate
PVA substrate

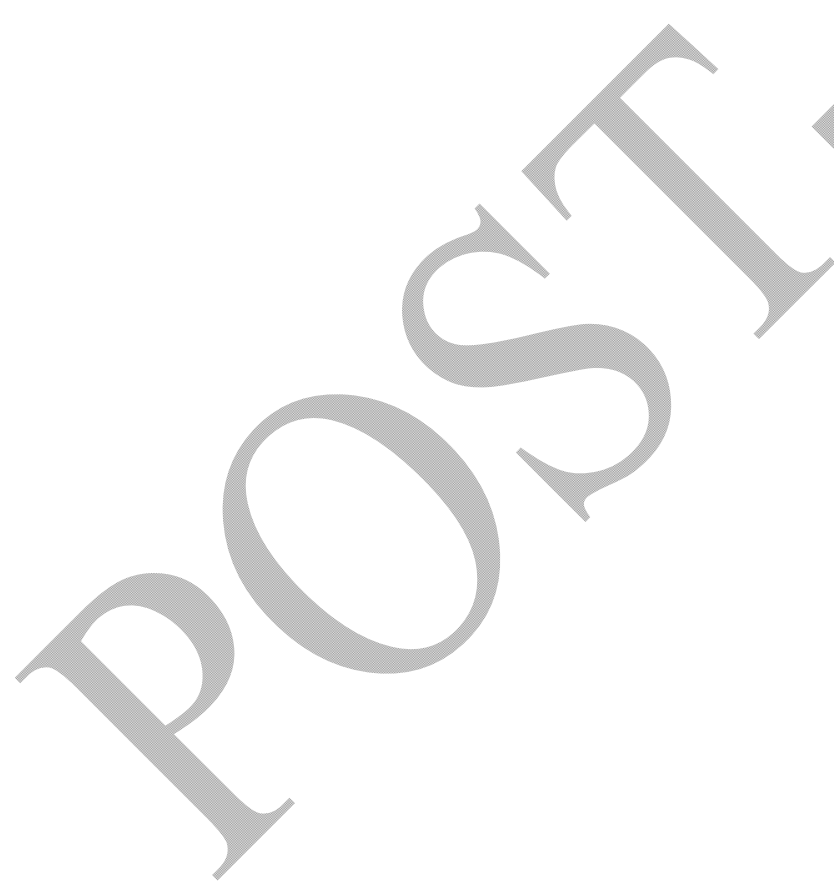


a

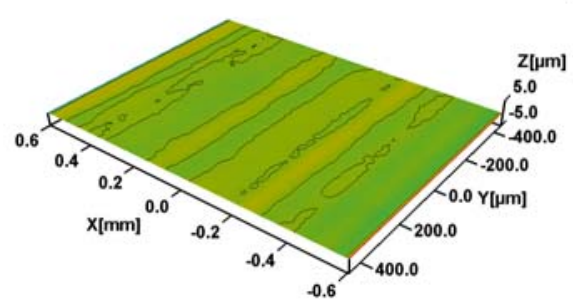

Si (Silicon Wafer)

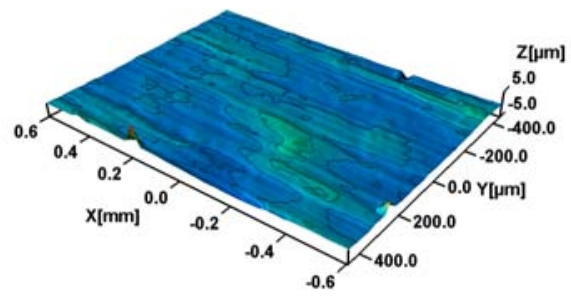

CM (Compression Molded)

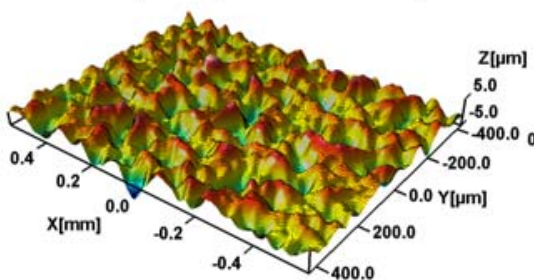

BP (Build Platform)

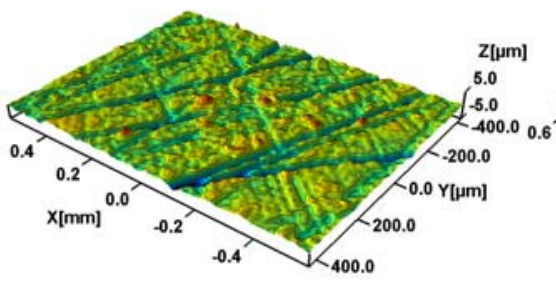

CM-S (Hand-roughened)

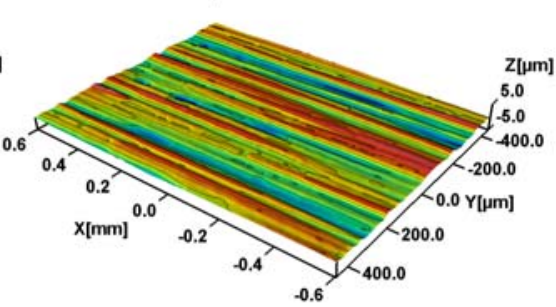

Al (Aluminum Chip)

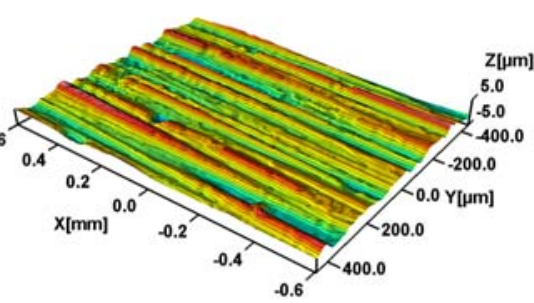

IM (Injection molded)

b Arithmetical Mean Height (Sa) - ISO 25178 (Area: 255 x $191 \mu \mathrm{m}$ )

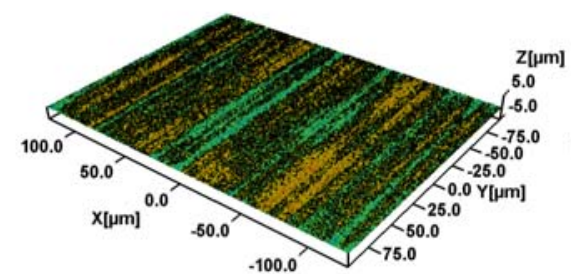

Si (Silicon Wafer)

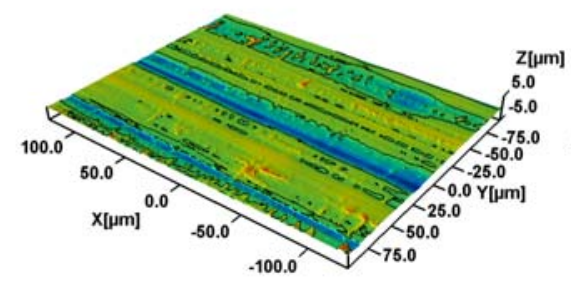

CM (Compression Molded)

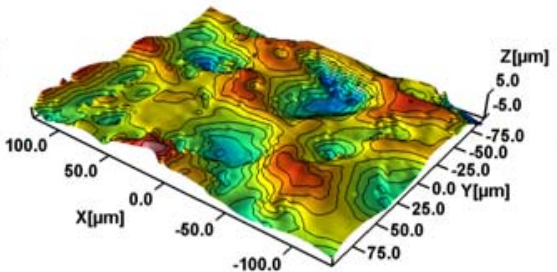

BP (Build Platform)

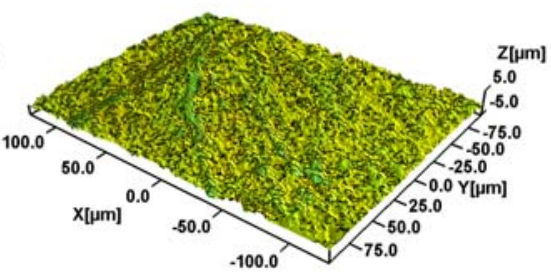

CM-S (Hand-roughened)

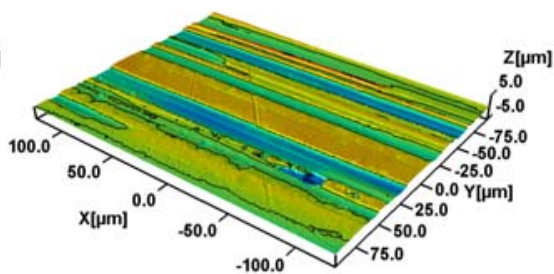

Al (Aluminum Chip)

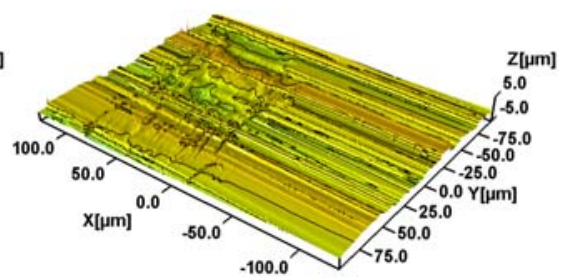

IM (Injection molded)

Figure 4. Flatness and roughness measurements obtained by optical profilometry. a)

500 Representative surface renderings of substrates used for flatness analysis. Computed from

501 data acquired with a $20 \mathrm{X}$ confocal lens in stitching mode (BP, CM and CM-S) and a 10X

502 interferometry lens (Si, Al and IM). b) Representative surface renderings of data used for

503 roughness analysis (Sa and $\mathrm{Sdr}$ ). Computed from acquisitions with a 50X interferometry lens. 
a

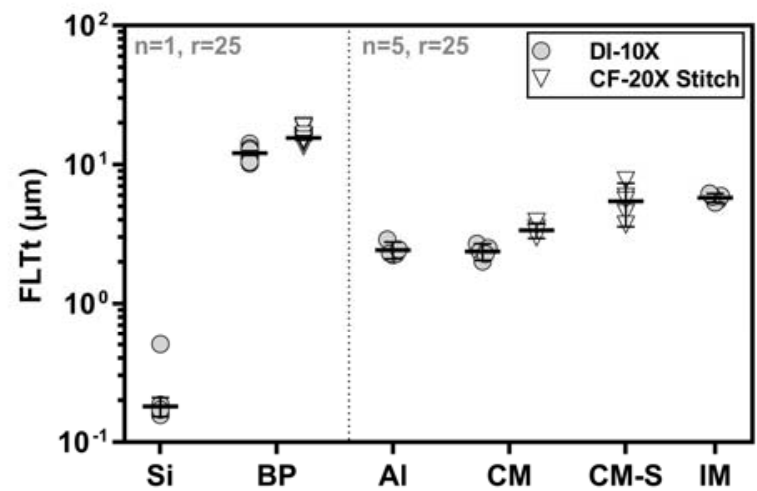

C

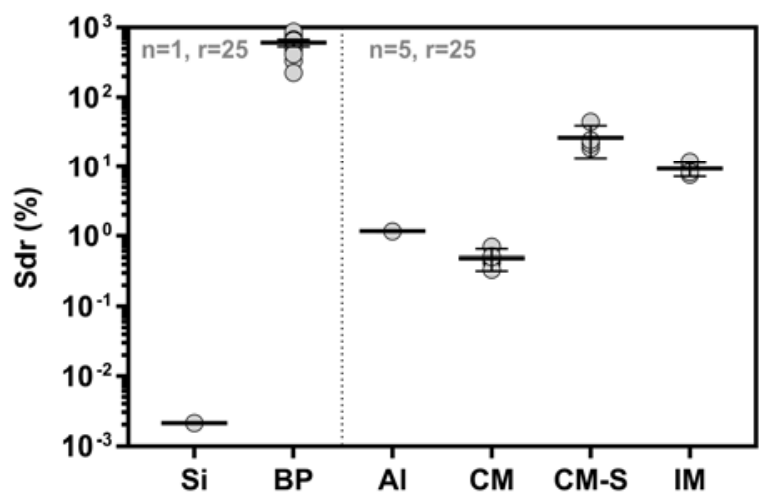

b

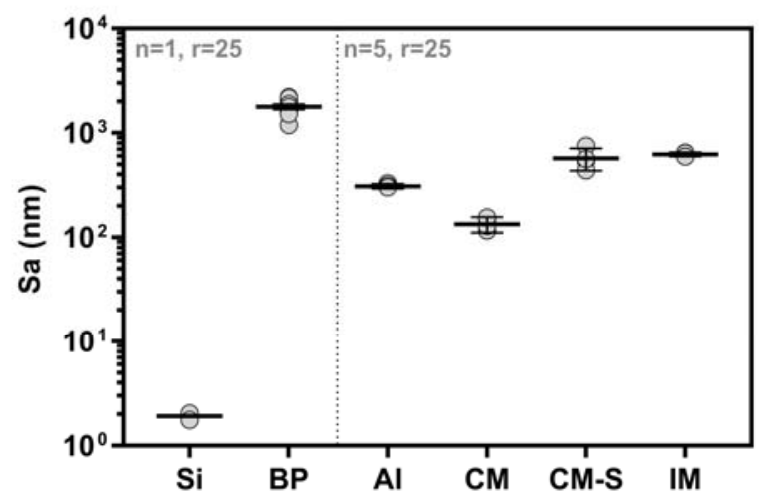

d

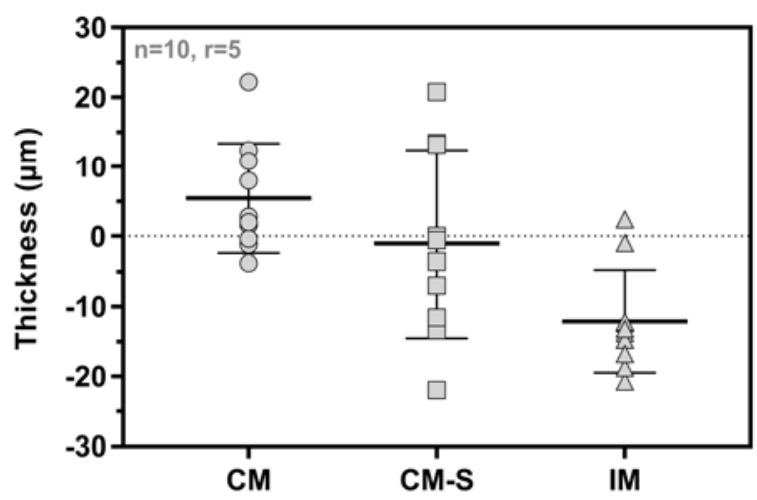

Figure 5. Geometrical characterization of different 3D printing substrates: Plain aluminum (Al), compression molded (CM), hand-roughened CM (CM-S) and injection molded (IM) PVA and reference substrates: Silicon wafer ( $\mathrm{Si}$ ) and commercial anodized 3D printer build platform (BP). Error bars represent 95\% confidence interval in a), b) and c) and standard deviation in d). a) Peak-to-valley flatness deviation (FLTt) measurements from optical profilometry surface data obtained with digital interferometry (DI) and confocal (CF) observation conditions. For statistical comparison see Table S 1. b) Arithmetical mean height (Sa) measurements from optical profilometry surface data. For statistical comparison see Table S 2. c) Developed interfacial area ratio (Sdr) computed from optical profilometry surface data. Statistical comparison available in Table S 3. For a), b) and c) counts: $\mathrm{N}=5$ with 5 different samples in case of $\mathrm{Al}, \mathrm{CM}, \mathrm{CM}-\mathrm{S}$ and IM and $\mathrm{N}=1$ with 25 repeated measurements on the same sample in case of Si and BP. d) Micrometer thickness measurements of PVA substrates adjusted to target values with $\mathrm{Y}=0=$ target thickness value. $\mathrm{N}=10$. 

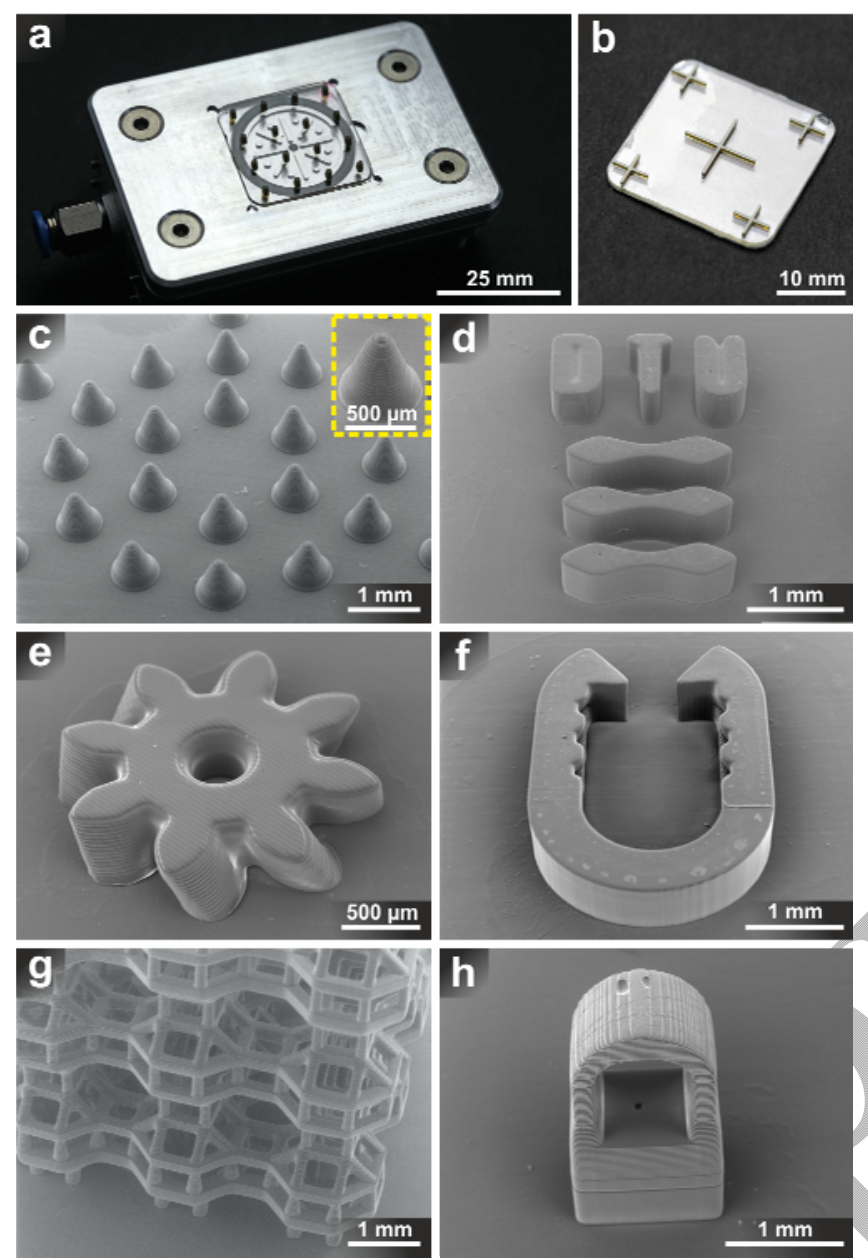

Figure 6. Photographs and SEM micrographs of 3D printed structures on compression molded PVA substrates (CM). a) Array of printed structures on PVA substrate inserted in vacuum-actuated holder (see schematics in Figure 1a and b). b) 3D printed crosshairs, facilitating evaluation of alignment of PVA substrate and printed structures. c) Circular array of micro-cones. d) DTU logo assembly from separate 3D printed parts. e) Helical micro-gear with a twist of $25^{\circ}$. f) Surgical staple. g) Complex lattice made from micro-sized trusses. ${ }^{[26]} \mathrm{h}$ ) Small structure used for evaluation of bond strength of 3D print to PVA substrate. 

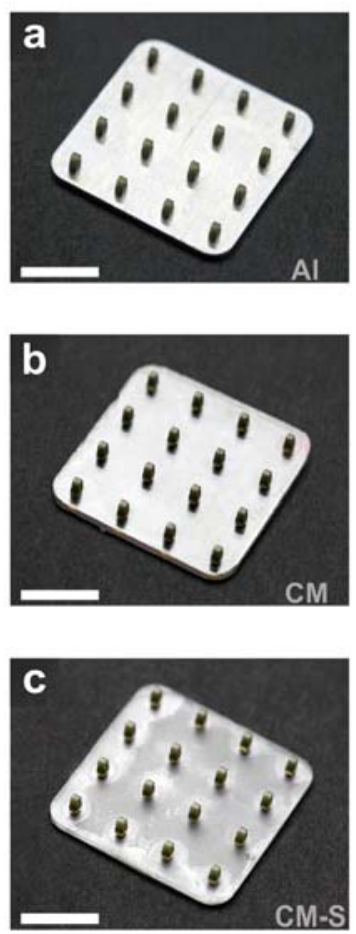

d

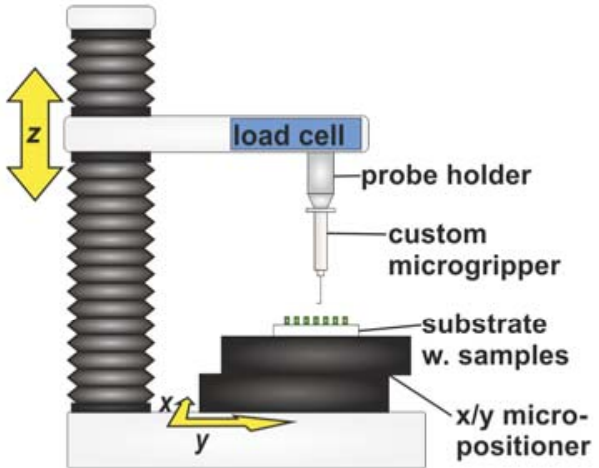

f

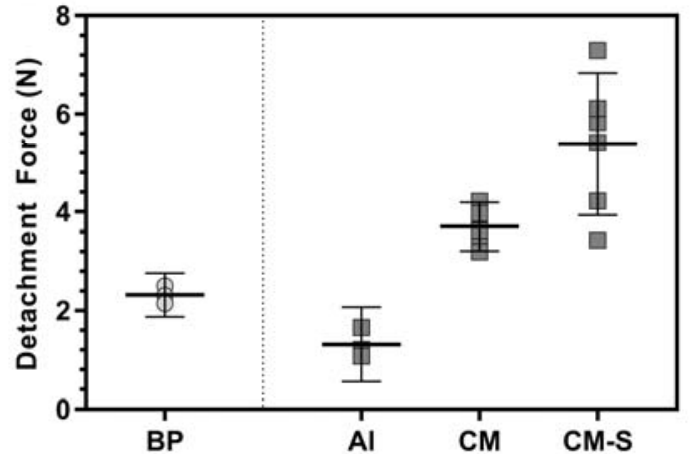

e

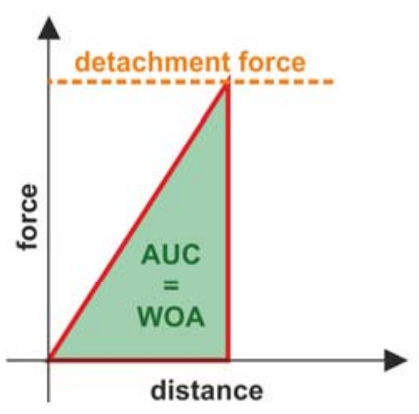

g

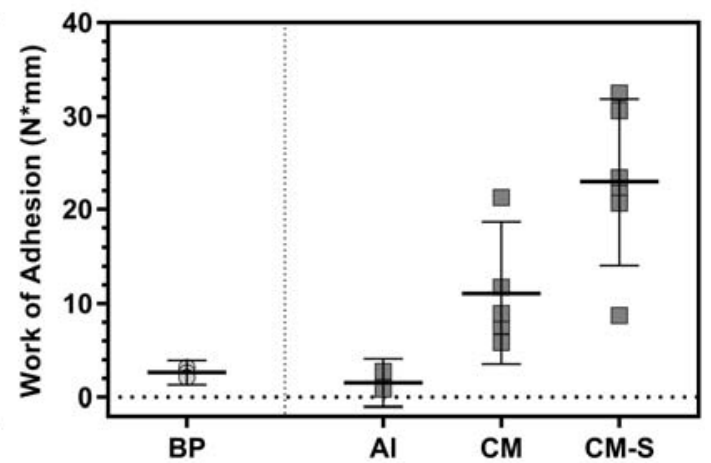

Figure 7 Determination of detachment forces/bond strengths of 3D printed objects on PVA substrates. a-c) Photographs of manufactured samples. Scale bars are equal to $10 \mathrm{~mm}$. a) Test structures 3D printed on plain aluminum substrates (Al). b) Test structures 3D printed on compression molded PVA substrates (CM) and c) hand-roughened CM PVA substrates (CMS). d) Schematic illustration of texture analyzer test-setup used for the experiments. e) Schematic illustration of obtained displacement curves. f) Evaluation of detachment forces. g) Determined work of adhesion (WOA), which is equal to the area under the curve (AUC) of the displacement graph. Additional to the manufactured samples, a commercial 3D printer build platform (BP) also served as reference substrate. $\mathrm{N}=3-6$. Error bars represent $95 \%$ confidence interval. Statistical evaluation available in Table S 4 and Table S 5. 

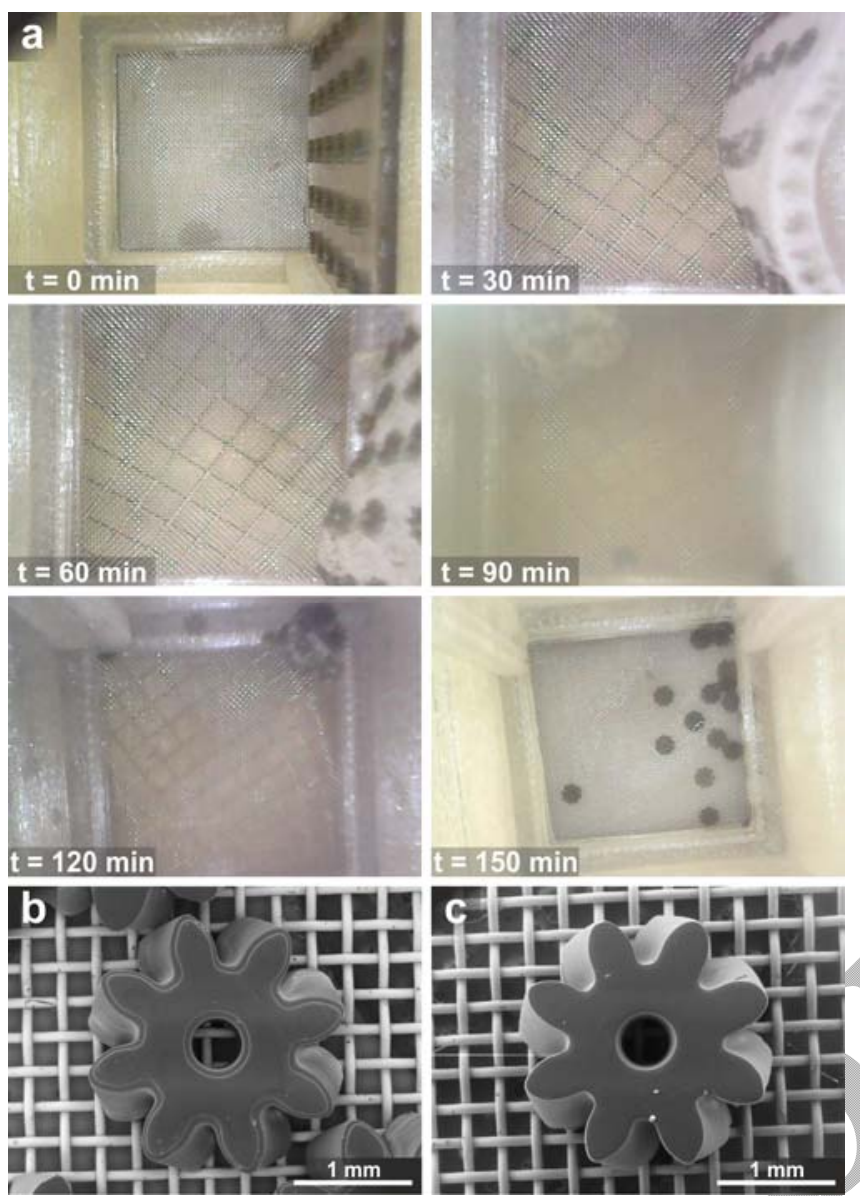

Figure 8. Release of 3D printed objects from PVA substrates. a) Time-lapse photos taken with a water-resistant endoscopic camera during the release procedure of $3 \mathrm{D}$ printed microgears from compression molded PVA substrates (CM). Release procedure was carried out in a customized release-chamber/substrate-holder combination at $55{ }^{\circ} \mathrm{C}$ in an ultrasound bath. b) and c) SEM micrographs of 3D printed helical micro-gears (see Figure 6e) on stainless steel filtering mesh after dissolution of compression molded PVA substrates (CM) and subsequent release. b) front side. c) backside. 
3D printing on top of sacrificial substrates is demonstrated. The used 3D printing workflow enables the 3D printing on quickly exchangeable substrates, further array-based processing of 3D printed products and easy manipulation, as well as integration into industrial production lines. 3D printed products can be mildly released from the substrates upon dissolution of sacrificial material and harvested.

\section{D printing}
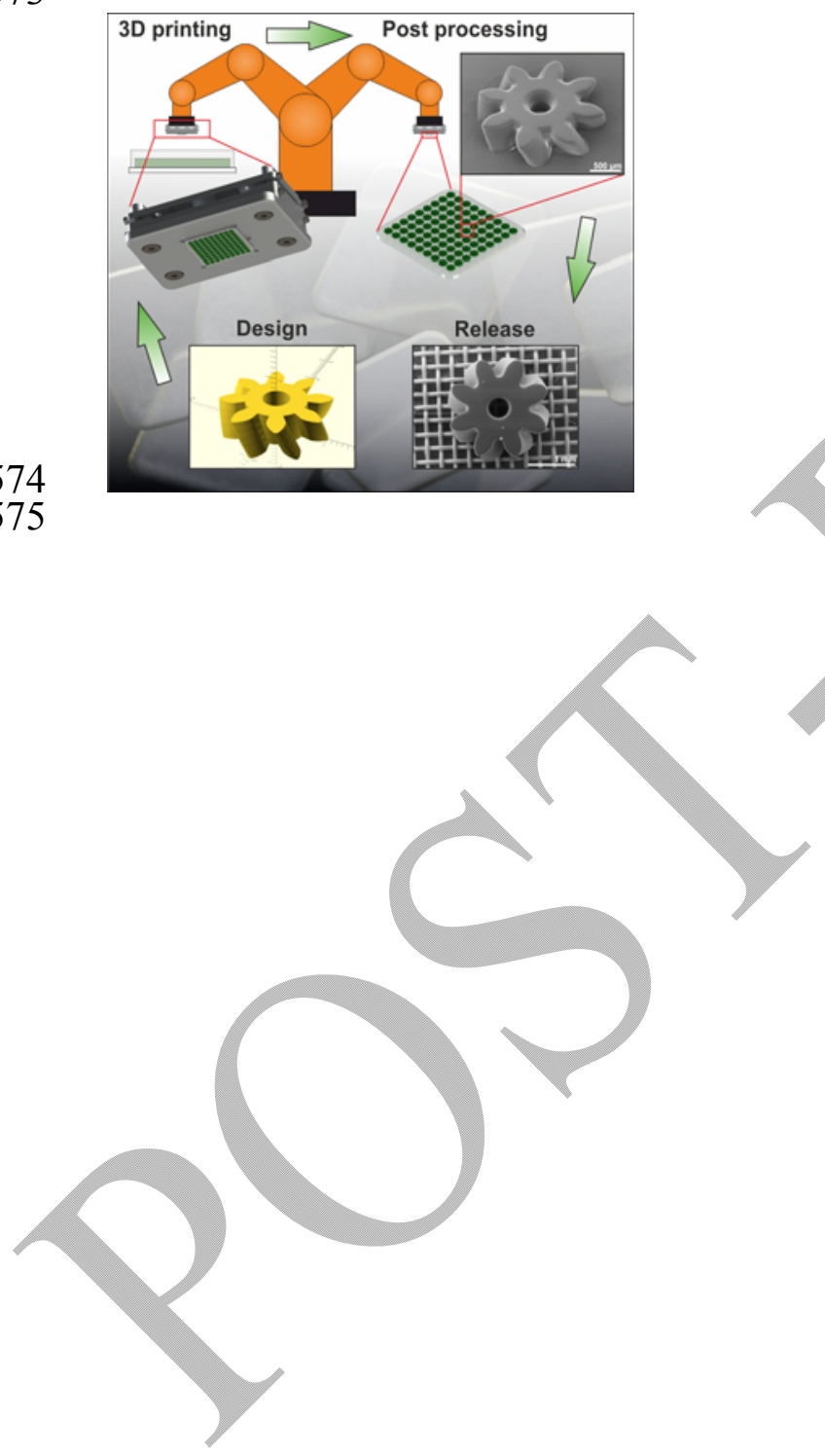